\title{
Design of Novel BSA/Hyaluronic Acid Nanodispersions for Transdermal Pharma Purposes
}

\author{
Madalena Martins, ${ }^{\dagger}$ Nuno G. Azoia, ${ }^{\dagger}$ Ulyana Shimanovich, ${ }^{\ddagger}$ Teresa Matamá, ${ }^{\S}$ Andreia C. Gomes, ${ }^{\S}$ \\ Carla Silva, ${ }^{\dagger, \perp}$ and Artur Cavaco-Paulo ${ }^{* \dagger}$ \\ ${ }^{\dagger}$ Centre of Biological Engineering, Universidade do Minho, Campus de Gualtar, 4710-057 Braga, Portugal \\ ${ }^{\ddagger}$ Department of Chemistry, University of Cambridge, Lensfield Road, Cambridge, CB2 1EW, United Kingdom \\ ${ }^{\S}$ CBMA (Centre of Molecular and Environmental Biology), Department of Biology, University of Minho, Campus of Gualtar, \\ 4710-057 Braga, Portugal
}

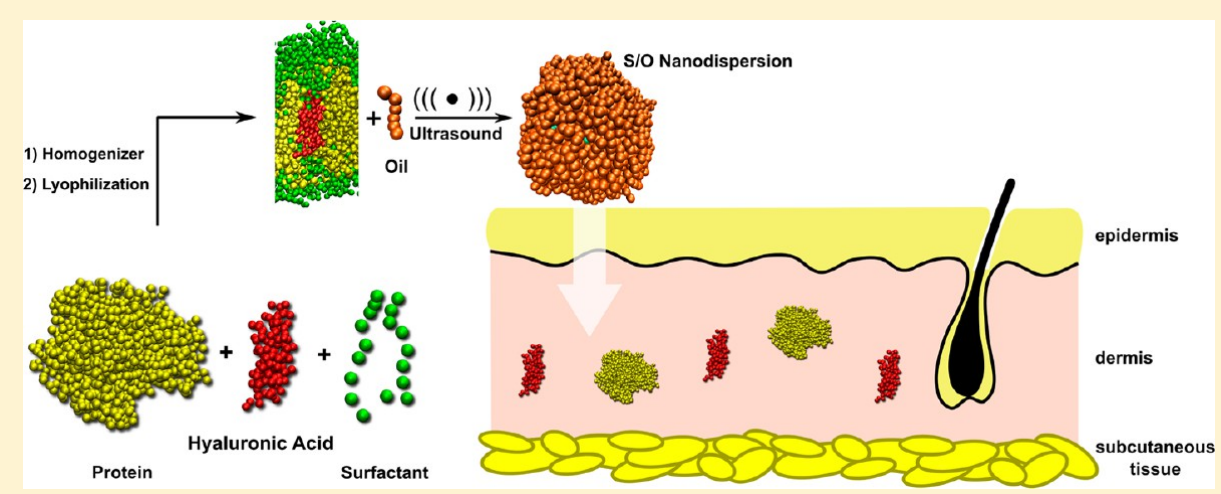

ABSTRACT: A novel transdermal hyaluronic acid (HA) conjugated with bovine serum albumin (BSA) was developed in the form of solid-in-oil (S/O) nanodispersion (129.7 nm mean diameter). Ex vivo skin penetration analysis by fluorescence and confocal observation of histological skin sections revealed the ability of BSA/HA nanodispersions to cross the stratum corneum and penetrate into the dermis. Furthermore, no significant toxicity was found in fibroblast and keratinocyte cells in vitro. These results proved the potential of the developed nanodispersion for transdermal delivery of hyaluronic acid constituting a high value to biopharmaceutical and cosmetics industries.

KEYWORDS: hyaluronic acid, bovine serum albumin, nanodispersion, transdermal transport, skin penetration

\section{INTRODUCTION}

Skin is the largest organ in the body, with the major function of protecting from dehydration and unwanted effects caused by the external environment. ${ }^{1}$ Aging is a dynamic process that may result from repeated chemical assault (e.g., soaps or cosmetics) or from a lifetime of exposure to UV radiation from sunlight. ${ }^{2}$ The presence of hyaluronic acid (HA) in pharmaceuticalcosmetic formulations would be able to protect the skin against these side effects. ${ }^{3}$ It is well-known that HA can reduce facial lines and wrinkles in the long term with fewer side effects and better tolerability compared with the use of collagen. ${ }^{4-6} \mathrm{HA}$ is present in the intercellular matrix of most vertebrate connective tissues, especially skin, where it has a protective, structure stabilizing, and shock-absorbing role. ${ }^{7} \mathrm{HA}$ is a naturally occurring biopolymer, polyanionic polysaccharide that consists of $\beta$-glucoronic acid and $\mathrm{N}$-acetyl-D-glucosamine with hydrophilic properties (a very high affinity for attracting and binding water molecules). ${ }^{8}$ Its molecular weight is strongly dependent on its source of commercial production $^{9}$ and varies between $10^{2}$ and $10^{4} \mathrm{kDa}$ in different tissues and fluids. ${ }^{10}$

Properties of HA including specific biocompatibility, nonimmunogenicity, biodegradability, viscoelasticity, hydration, and lubrication ${ }^{11,12}$ make this polysaccharide potentially useful in food, medical, pharmaceutical, and cosmetic industries.

HA controls tissue hydration and exists in hydrated networks with collagen fibers in the intercellular matrix. It also constitutes the backbone of cartilage proteoglycan. Because of its unique physicochemical properties and various biological functions, HA and modified HA have been extensively investigated and widely used for pharmaceutical and medical applications, namely, as a novel drug carrier for target specific and long-acting delivery of proteins, peptides, and nucleotide therapeutics. ${ }^{13}$ In contrast, cosmetic and food applications have received little attention. ${ }^{14}$

Hydrophilic macromolecules, like HA, constitute a challenging issue in the development of transdermal transport systems to cross the intact skin and reach the lipid bilayers. ${ }^{15,16}$ Transdermal transport systems have many advantages over other systems because they can promote effective patient compliance without painful injections. ${ }^{17,18}$ Despite these benefits, the low bioavailability

Received: November 4, 2013

Revised: February 27, 2014

Accepted: March 20, 2014

Published: March 20, 2014 
constitutes the major disadvantage of transdermal transport systems due to poor skin permeability. This is mainly due to the stratum corneum, the outermost layer of skin, which forms a barrier based on epidermal lipids. This fact allows transport of compounds only with low molecular weight $\left(M_{\mathrm{w}}\right)(<500 \mathrm{Da}) \cdot{ }^{19-21}$ The barrier permeability provided evidence for the crucial nature of the three major lipid species: cholesterol, ceramides, and free fatty acids. ${ }^{22,23}$ Thus, as the decisive role of skin care, strategies focus on restoring, repairing, or protecting the barrier skin by application of selected lipids for improving skin function and appearance. ${ }^{24}$ To circumvent the limitation of transporting hydrophilic macromolecules across the skin layers, a S/O dispersion technology was designed assuming the formation of an external layer of a hydrophobic moiety. ${ }^{15}$ This technology was developed as a novel platform for oral and skin drug delivery systems. ${ }^{25}$

In our previous study, a large protein, BSA (66 kDa), was coated with hydrophobic surfactant molecules using an adapted and optimized S/O methodology. ${ }^{26}$ A formulation containing small dispersions (160 nm mean diameter) that successfully cross the skin barriers was achieved. ${ }^{26}$

The major delivery method for biopharmaceutics to human body is currently done via subcutaneous or intravenous administration, in particular for rejuvenative medicine as a filler for cutaneous lines and wrinkles. ${ }^{2,28}$ The potential of HA for transdermal delivery has been reported as a promising drug carrier. ${ }^{13,14,29}$ There is no pharmaceutical/cosmetic formulation study available using HA in the form of S/O nanodispersion exhibiting a good permeability through all skin layers. Herein, transdermal capacity and bioavailability of BSA/HA solid-in-oil nanodispersions with different molecular weight $\mathrm{HA}$ were investigated. In vitro permeation profile into pig skin was studied quantitatively and qualitatively.

\section{EXPERIMENTAL SECTION}

2.1. Materials. BSA-FITC, chloroform, and isopropyl myristate were commercially supplied by Sigma-Aldrich and used as received. Sucrose ester (SE), Sisterna SP10-C, was purchased from Sisterna (Sisterna, The Netherlands). Hyaluronic acid of molecular weight $\left(M_{\mathrm{w}}\right) 800 \mathrm{kDa}$ from cocks comb was supplied by Sigma Aldrich. Hyaluronic acid (50 and $15 \mathrm{kDa}$ ) was kindly provided by Lifecore (Lifecore, USA) and (HA $100 \leq$ $M_{\mathrm{w}} \leq 300$ and $20 \leq M_{\mathrm{w}} \leq 50 \mathrm{kDa}$ ) from Soliance (Soliance, France), both from bacterial source. The products used for histology procedure were supplied by Fisher Healthcare (U.K.). The transdermal studies were performed using pig skin from a slaughterhouse and kindly supplied by Matadouro Central de Entre Douro e Minho (Portugal). For histology studies the Cryoprotectant Tissue-Tek Oct compound was purchased from Sakura (The Netherlands) and the Thermo Scientific Shandon Immu-Mount was purchased from Fisher Healthcare (U.K.). All other reagents were laboratory grade without any further purification.

2.2. Preparation of $S / O$ BSA Nanodispersions Containing Hyaluronic Acid. The S/O protein/polymer (BSA-FITC/HA) nanodispersions were prepared by an adaptation of our previous method. ${ }^{26}$ Briefly, the initial complex between sucrose ester (SE) surfactant and protein (mixed with hyaluronic acid) was formed by high pressure homogenization at 1000 bar (EmulsiFlex-C3, Avestin, Canada). For this, the concentrations of BSA-FITC used were $0.1,0.25,0.5$, and $1 \mathrm{mg} / \mathrm{mL}$, mixed with several amounts of hyaluronic acid, namely, $0.003,0.006$, and $0.009 \mathrm{mg} / \mathrm{mL}$, for the different HA molecular weights tested. For each formulation a fixed amount, $25 \mathrm{mg} / \mathrm{mL}$, of SE surfactant (SP10-C) was applied.
Then a lyophilization step was performed aiming to eliminate water and chloroform (solvent used for surfactant solubilization) attaining a solid surfactant-protein complex. This complex was dispersed in oil, isopropyl myristate (IPM), by ultrasonication using an ultrasound probe $(20 \mathrm{kHz}$ Sonics \& Materials Vibracell CV33) fitted with a $3 \mathrm{~mm}$ diameter titanium microtip, forming the final $\mathrm{S} / \mathrm{O}$ nanodispersion.

2.3. S/O Nanodispersion Characterization. 2.3.1. Determination of Production Yield. The nanodispersion production yield was determined by centrifugation $(15000 \mathrm{~g}, 30 \mathrm{~min}$ ) and separation of the formulated part from the aqueous preparation medium containing the nonformulated protein. The amount of free BSA-FITC was determined in the supernatant by the Lowry method protein assay (Sigma), measuring the absorbance by spectrophotometry (Microplate Reader Synergy MX, Bioteck Instruments) at $750 \mathrm{~nm} .{ }^{30}$ Each sample was assayed in triplicate $(n=3)$. The concentration of protein was determined by a calibration curve. The S/O nanodispersion production yield (PY) was calculated as follows:

$$
\mathrm{PY}(\%)=\frac{[\text { protein }]_{\mathrm{i}}-[\text { protein }]_{\text {supernatant }}}{[\text { protein }]_{\mathrm{i}}} \times 100
$$

2.3.2. Assembly Efficiency of Hyaluronic Acid. Assembly efficiency (AE) of hyaluronic acid in BSA-FITC solid-in-oil nanodispersions was determined by their separation from the aqueous preparation medium containing the free hyaluronic acid by centrifugation $(2000 \mathrm{~g}, 30 \mathrm{~min})$ using Centricon tubes (molecular weight cutoff $1000 \mathrm{kDa}$ ). The amount of free hyaluronic acid was determined in the supernatant by UV spectrophotometry, using Microplate Reader Synergy MX (Bioteck Instruments) at $185 \mathrm{~nm}$.

$$
\mathrm{AE}(\%)=\frac{[\mathrm{HA}]_{\mathrm{i}}-[\mathrm{HA}]_{\mathrm{f}}}{[\mathrm{HA}]_{\mathrm{i}}} \times 100
$$

$[\mathrm{HA}]_{\mathrm{i}}$ is the initial concentration of hyaluronic acid used, and $[\mathrm{HA}]_{\mathrm{f}}$ is the final concentration of hyaluronic acid in supernatant.

2.3.3. Determination of $z$-Average Diameter. The $z$-average diameter of nanodispersions was determined at $25 \pm 0.1{ }^{\circ} \mathrm{C}$ using a Malvern Zetasizer NS (Malvern Instruments) by photon correlation spectroscopy (PCS). For each nanodispersion formulation the average diameter was measured in triplicate.

2.3.4. S/O Nanodispersion Morphology: Transmission Electron Microscopy (TEM). The morphological characterization of nanodispersions was conducted using transmission electron microscopy technique in an electron microscope model LEICA S360 with a backscattered and secondary electron detector, at $50000 \times$ magnification. The samples were placed on copper grids with carbon film for TEM observation.

2.3.5. S/O Nanodispersion Stability. The physicochemical stability of nanodispersions was assessed taking into account their storage stability. The stability was evaluated by size distribution and polydispersity index (PDI). The formulations were stored at room temperature over 3 months.

The biological stability of nanodispersions by oxidative evaluation was also performed. For that, the lipid peroxidation experimental protocol was used. The detection of lipid peroxidation was assessed using a spectrophotometrical technique to measure one of the secondary products formed and used to estimate lipid peroxidation, the malondialdehyde (MDA), after the reaction with thiobarbituric acid. ${ }^{31}$

2.4. In Vitro Permeation Studies through Pig Skin. Ex vivo study of permeation through full thickness pig skin was 
achieved using the horizontal Franz diffusion cell (V-Series Stirrers for Franz Cells) (PermeGear, USA). ${ }^{26}$ The pig skin specimens were provided by a local slaughterhouse. First, the skin was removed from the pig without any steam cleaning. Then, the subcutaneous fat was maximally removed and was cut to an appropriate size and immediately mounted in the Franz diffusion cell, with the SC side facing the donor compartment. The receptor compartment was filled with $5 \mathrm{~mL}$ of PBS solution (phosphate buffered saline $0.01 \mathrm{M}, \mathrm{pH} 7.4$ ), which was continuously stirred with a small magnetic bar and thermostated at $37 \pm 1{ }^{\circ} \mathrm{C}$ throughout the experiments to reach the physiological skin temperature (i.e., $32 \pm 1{ }^{\circ} \mathrm{C}$ ). The amount of the S/O nanodispersion applied to the skin surface was approximately $300 \mu \mathrm{L}$. At regular intervals of $0,2,4,6,8,24,48$, and $72 \mathrm{~h}$, samples of the receiving solution were collected. Each sample removed was replaced with fresh buffer. To evaluate the transdermal permeation of $\mathrm{S} / \mathrm{O}$ nanodispersion, the protein concentration was quantified using the Lowry assay on the supernatant on each sample removed in the predetermined intervals. For the detection of HA, concentration was performed by monitoring the UV absorbance at $185 \mathrm{~nm}$. Controls were performed for all time points without protein and HA.

2.4.1. Fluorescence and Confocal Microscopy Studies. In order to evaluate the penetration of nanodispersions through the skin, fluorescent and confocal laser scanning microscopy was performed after $72 \mathrm{~h}$, the total time of the in vitro penetration experiment. For imaging purposes, BSA and HA were labeled with FITC (fluorescein isocyanate) and RITC (Rhodamine isocyanate), respectively. Skin samples were visualized by fluorescence microscopy taking into account different approaches to generate a phase contrast and differential interference contrast microscopy analysis. These two contrasting optical microscopy methods give very different but complementary data. Additionally, skin samples were optically scanned at different increments through the $Z$-axis of a confocal laser microscope (Zeiss LSM 510). The image reconstructions were made using "Imaris" image analysis software.
2.5. Cytotoxicity Evaluation. The nanodispersions of BSA/ HA were evaluated by one of the mostly used cytotoxicity or cell proliferation assays, the MTT, which is a quantitative colorimetric assay. In this assay, the yellow tetrazolium salt MTT (3-[4,5dimethylthiazol-2-yl]-2,5-diphenyl tetrazolium bromide) is reduced by living cells to blue formazan crystals, which must be solubilized in a solvent, such as isopropanol. ${ }^{32}$ The MTT assay appeared to be a sensitive test which shows linearity over a broad range of cell densities. ${ }^{32,33}$

The BJ5ta cell line (normal human skin fibroblasts) was maintained according to ATCC recommendations (4 parts Dulbecco's modified Eagle's medium (DMEM) with 1 part of Medium 199, 10\% (v/v) of fetal bovine serum (FBS), 1\% (v/v) of penicillin/streptomycin solution, and $0.01 \mathrm{mg} / \mathrm{mL}$ hygromycin B). The cells were maintained at $37{ }^{\circ} \mathrm{C}$ in a humidified atmosphere of $5 \% \mathrm{CO}_{2}$. Culture medium was refreshed every 2-3 days. Cells were seeded at a density of 80000 cells/well on 24-well tissue culture polystyrene plates (TPP, Switzerland), on the day before experiments, and then exposed to different nanodispersion formulation concentrations in a final volume of $0.5 \mathrm{~mL}$. Several controls were performed simultaneously: the death control with $15 \%$ (v/v) dimethyl sulfoxide (DMSO), the $100 \%$ viability control with just fresh medium, and a maximum dilution control with $50 \%(\mathrm{v} / \mathrm{v})$ phosphate buffered saline (PBS) to evaluate the effect of medium dilution. At 24 and $48 \mathrm{~h}$ of exposure, cell viability was determined using the MTT assay. $^{34-37}$

The human keratinocyte cell line NCTC 2544 was cultured in DMEM supplemented with 7\% (v/v) FBS and 1\% (v/v) of penicillin/streptomycin solution. Culture medium was refreshed every 2-3 days. Cells were seeded at a density of 45000 cells/well on 24-well tissue culture polystyrene plates (TPP, Switzerland), the day before experiments. The subsequent procedure was the same as that described for the BJ5ta line.

The cytotoxicity was evaluated by 3-(4,5-dimethylthiazol2-yl)-2,5-diphenyltetrazolium bromide (MTT) assay. In the MTT reduction assay the medium containing different dilutions of the

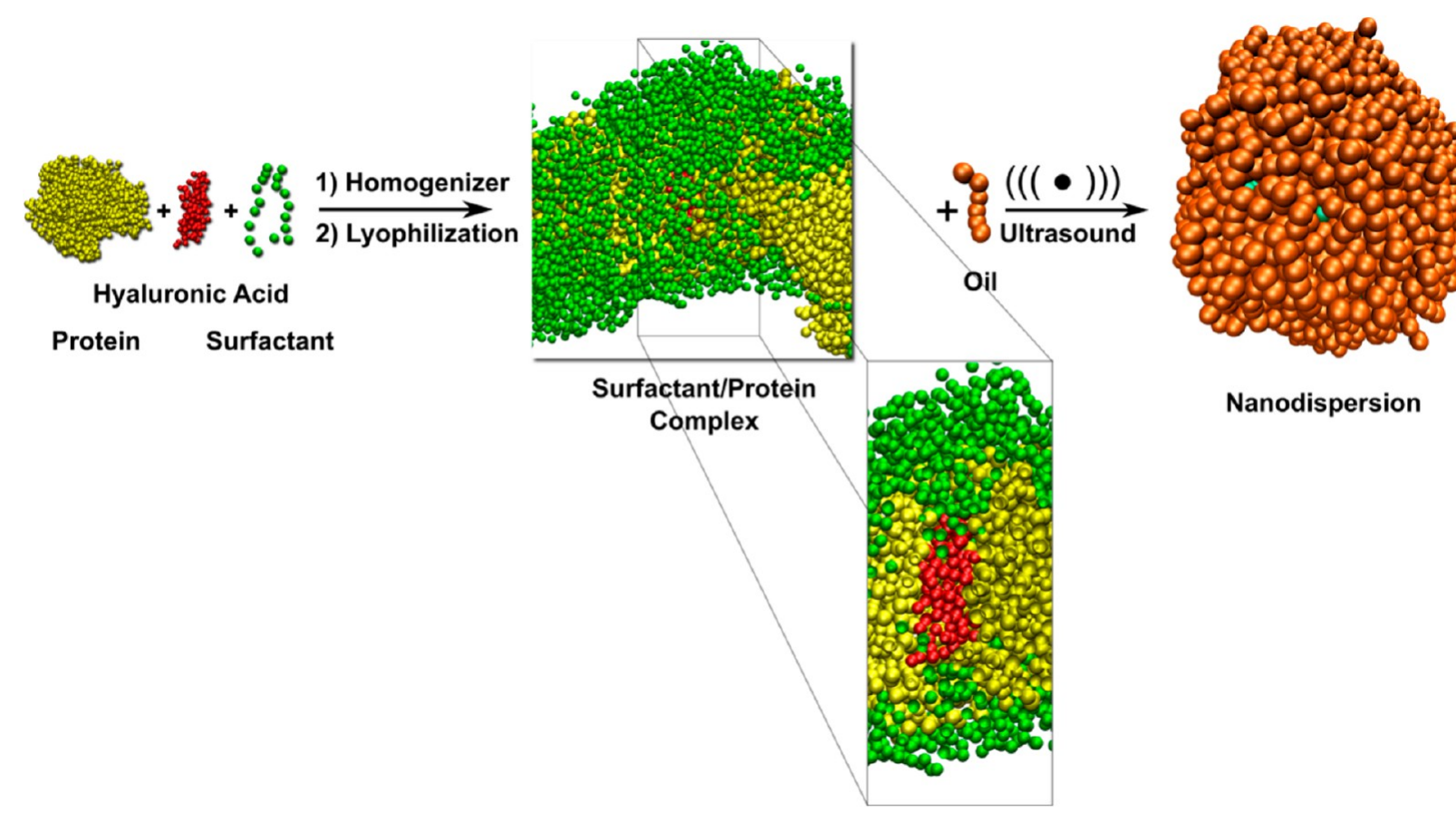

Figure 1. Schematic overview of the preparation method of S/O BSA/HA nanodispersions. 


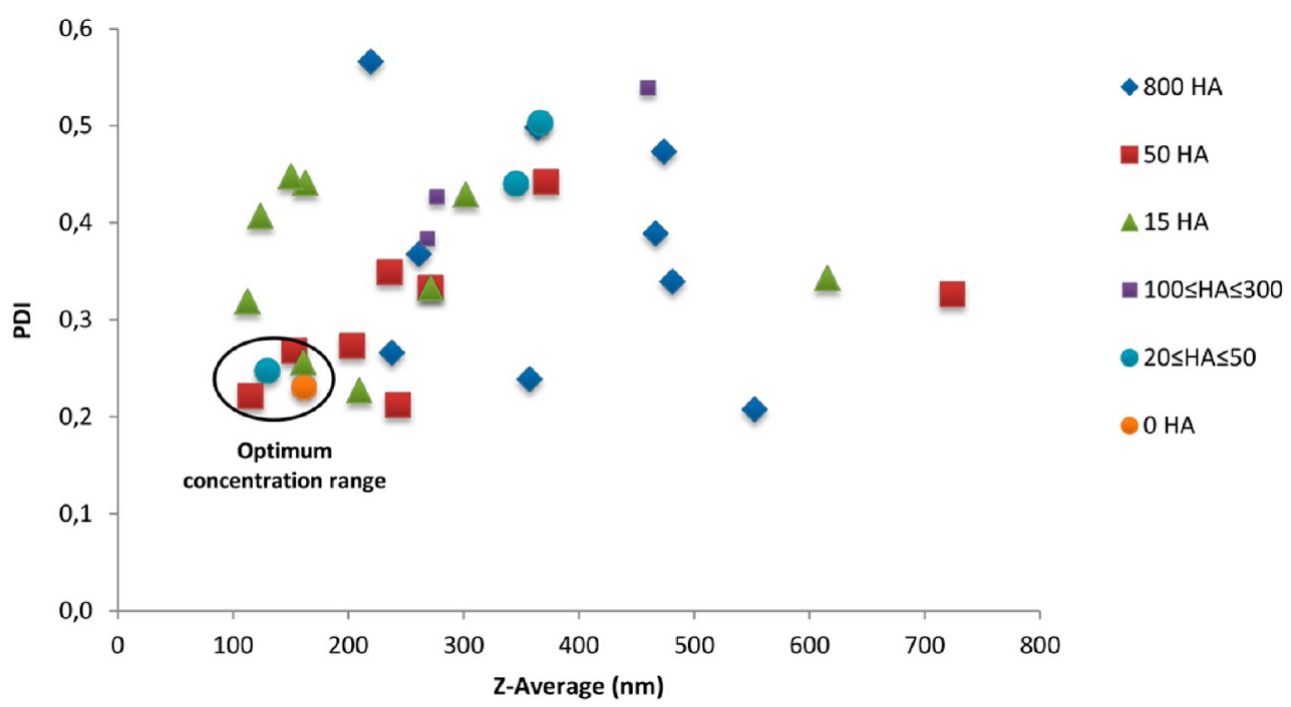

Figure 2. Evaluation of $z$-average $(\mathrm{nm})$ and PDI (mean $\pm \mathrm{SD}, n=3)$ of $\mathrm{S} / \mathrm{O}$ nanodispersions varying the amount of BSA mixed with several concentrations of different molecular weight of HA. Optimal particle characteristics in terms of size are lower than $200 \mathrm{~nm}$ and PDI lower than 0.280 . Their composition corresponds to $0.5 \mathrm{mg} / \mathrm{mL}$ of BSA with $0.006 \mathrm{mg} / \mathrm{mL}$ of HA for different molecular weight of HA; and for BSA formulation without $\mathrm{HA}, 1 \mathrm{mg} / \mathrm{mL}$.

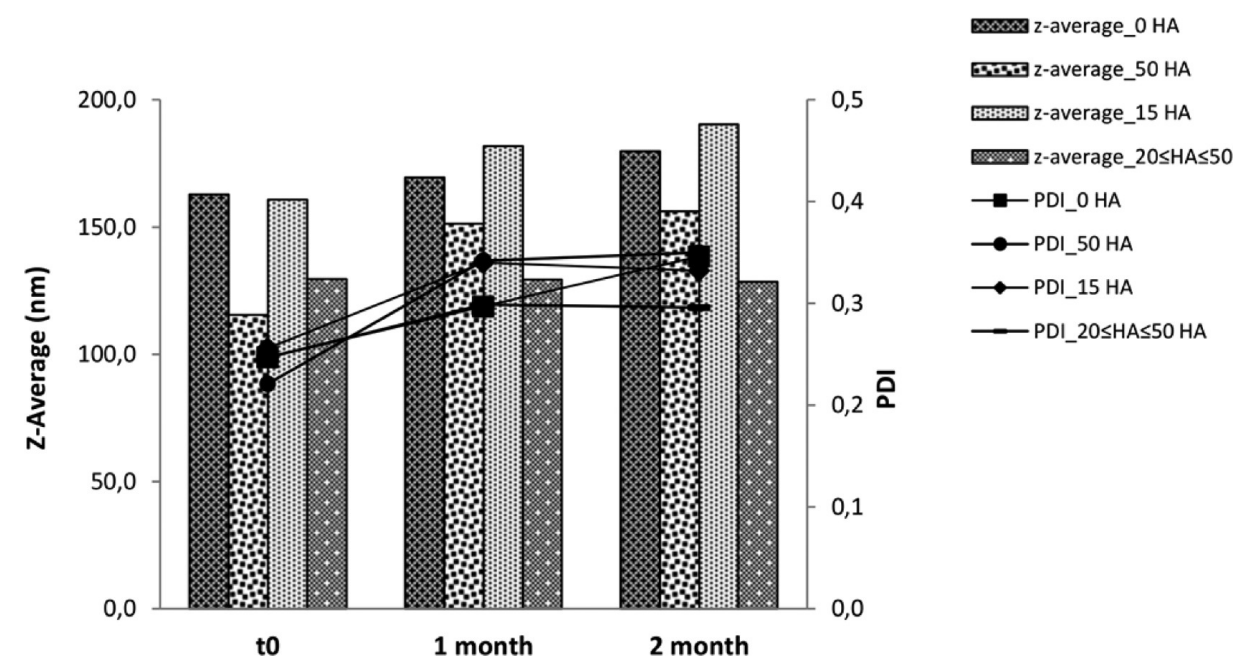

Figure 3. Stability of produced nanodispersions over time (2 months); for each sample the $z$-average and PDI were measured in triplicate.

Table 1. Physicochemical Properties, Production Yield, and Assembly Efficiency of S/O BSA/HA Nanodispersions Prepared with Different Molecular Weight Hyaluronic Acid (Mean \pm SD, $n=3$ )

\begin{tabular}{lcllllll}
\multicolumn{1}{c}{ samples } & BSA $(\mathrm{mg} / \mathrm{mL})$ & $\mathrm{HA} M_{\mathrm{w}}(\mathrm{kDa})$ & $\mathrm{HA}(\mathrm{mg} / \mathrm{mL})$ & size av $(\mathrm{nm})$ & PDI & PY $(\%)$ & AE $(\%)$ \\
BSA-FITC & 1 & & & $163.0 \pm 5.0$ & $0.247 \pm 0.006$ & $81.6 \pm 0.015$ & \\
A & 0.1 & 800 & 0.006 & $237.7 \pm 14.2$ & $0.266 \pm 0.04$ & $70.9 \pm 0.001$ & $90.6 \pm 0.028$ \\
B & 0.5 & 50 & 0.006 & $115.5 \pm 1.1$ & $0.221 \pm 0.02$ & $75.5 \pm 0.153$ & $89.8 \pm 0.042$ \\
C & 0.5 & 15 & 0.006 & $160.9 \pm 1.7$ & $0.256 \pm 0.005$ & $70.8 \pm 0.144$ & $89.6 \pm 0.008$ \\
D & 0.5 & $20 \leq M_{\mathrm{w}} \leq 50$ & 0.006 & $129.7 \pm 24.9$ & $0.247 \pm 0.04$ & $77.2 \pm 0.079$ & $90.8 \pm 0.095$ \\
\hline
\end{tabular}

nanodispersion formulation was removed, the wells were washed with warm PBS, and $0.5 \mathrm{~mL}$ of $0.5 \mathrm{mg} / \mathrm{mL} \mathrm{MTT} \mathrm{solution} \mathrm{was}$ added to each well. After incubation for $2 \mathrm{~h}$ at $37^{\circ} \mathrm{C}$, the medium was removed and the formazan crystals were solubilized in $0.5 \mathrm{~mL}$ of DMSO/ethanol solution. The plate was shaken for $5 \mathrm{~min}$ on a plate shaker, and the absorbance was measured at $570 \mathrm{~nm}$ in a microplate reader (Spectramax 340PC, Molecular Devices, Sunnyvale, CA, U.S.A.). The absorbance is directly proportional to the number of viable cells. The procedure was performed at least three times with duplicates, and the average values are presented.
2.6. Statistical Analysis. Statistical analysis of the experimental results was performed using analysis of variance (ANOVA). Differences were considered statistically significant at $p<0.05$. All data values are represented as mean \pm standard deviation of at least 3 measurements.

\section{RESULTS}

3.1. Preparation and Characterization of S/O Nanodispersions of BSA/HA. The $\mathrm{S} / \mathrm{O}$ nanodispersion containing the conjugate $\mathrm{BSA} / \mathrm{HA}$ was obtained by coating the $\mathrm{BSA} / \mathrm{HA}$ 

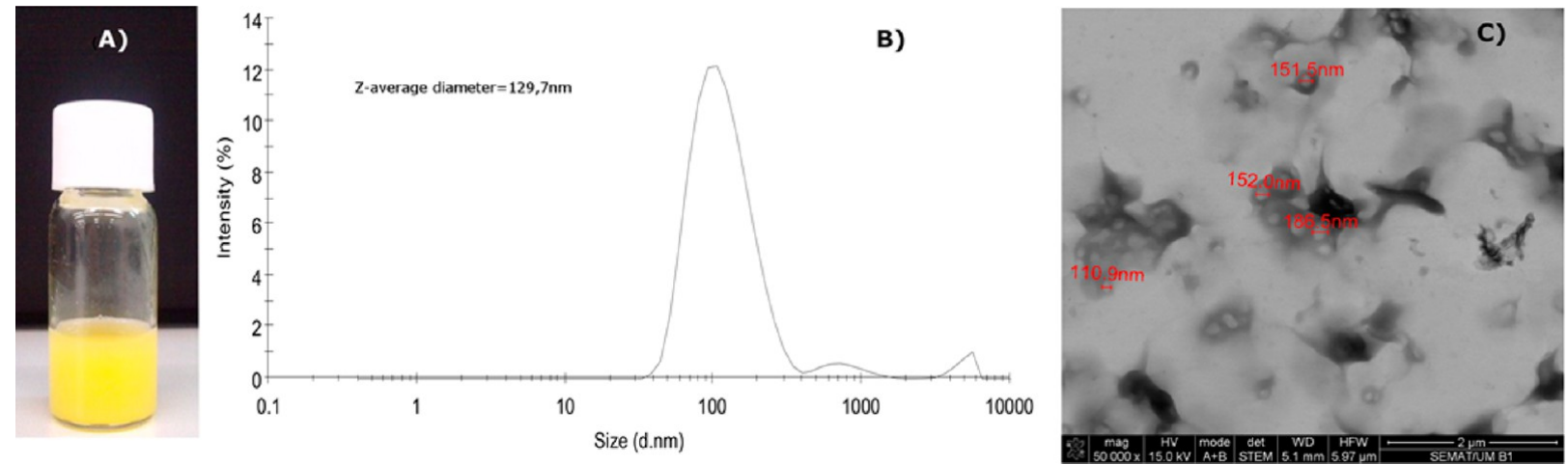

Figure 4. Illustration of S/O nanodispersion of BSA/HA: (A) physical appearance, (B) size distribution obtained by PCS (photon correlation spectroscopy), and (C) TEM microphotographs at magnitude 50000×.
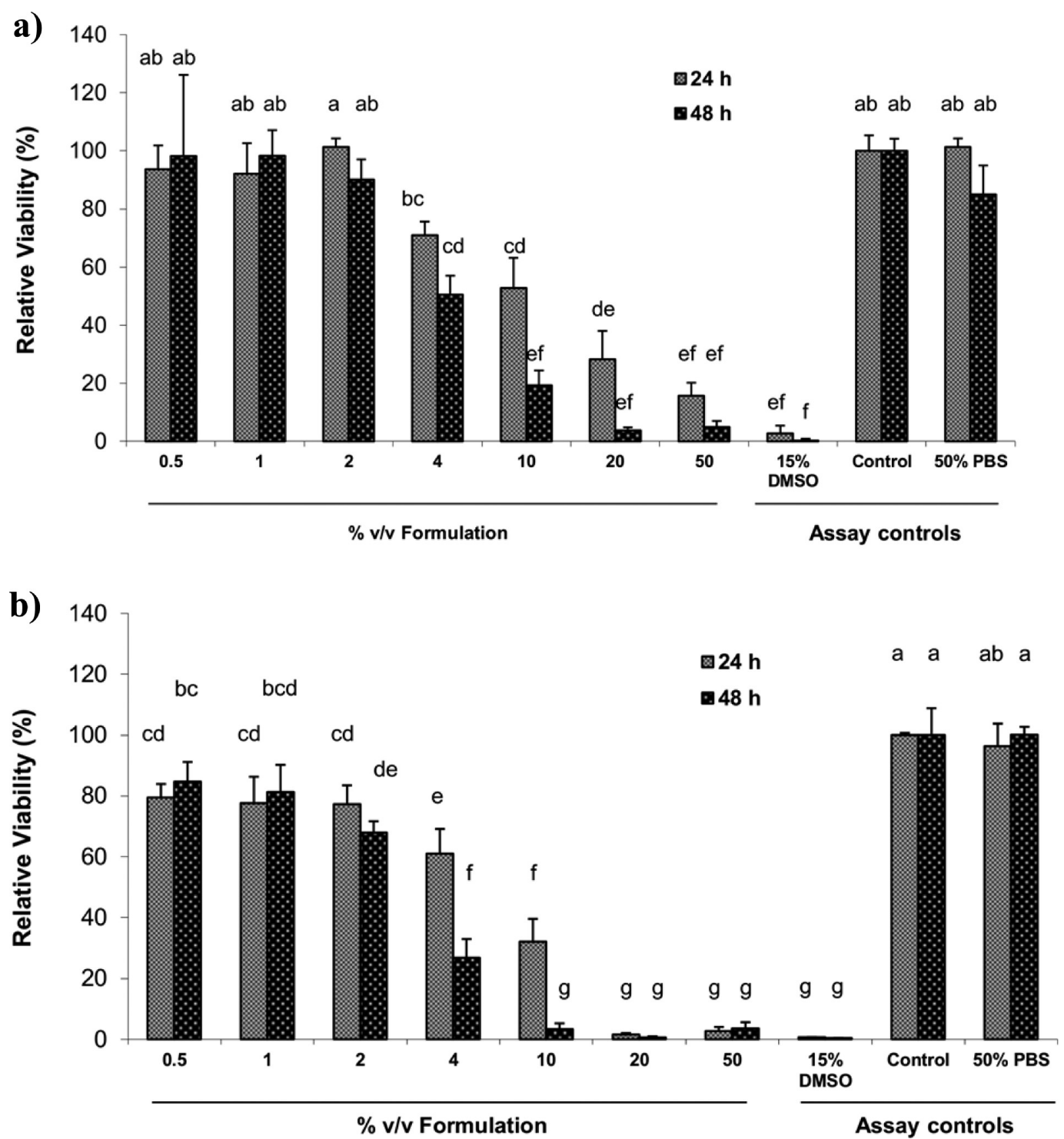

Figure 5. (a) BJ-5ta and (b) NCTC2544 relative cell viability at 24 and $48 \mathrm{~h}$ of culture for S/O nanodispersion of BSA $(0.5 \mathrm{mg} / \mathrm{mL}) \mathrm{mixed}$ with $\mathrm{HA}$ $(0.006 \mathrm{mg} / \mathrm{mL})$ at different dilutions $(\% \mathrm{v} / \mathrm{v}$ formulation). Values for tested samples are presented in relation to the control (cells cultured with culture medium scored $100 \%$ of viability). Results show the mean values of two replicates from four experiments carried out independently. Grouping information using Tukey test with $95 \%$ of confidence: means that do not share a letter are significantly different.

with a lipophilic surfactant (SE). This complex was then dispersed in oil to obtain the final S/O BSA/HA nanodispersion (Figure 1).
Recently, we have demonstrated that one sucrose ester, the surfactant SP10-C, has the potential to form stable complexes with BSA. ${ }^{26}$ The ability of this surfactant to produce small and 
stable nanodispersions in oil was a good starting point for investigating the best ratio among SE:BSA:HA for the production of nanodispersions containing hyaluronic acid. In order to achieve the optimal molar ration between all the components of the system, we mixed BSA-FITC $(66 \mathrm{kDa})$ with a wide range of molecular weight $\mathrm{HA}\left(800,100 \leq M_{\mathrm{w}} \leq 300,50\right.$, $20 \leq M_{\mathrm{w}} \leq 50$, and $\left.15 \mathrm{kDa}\right)$.

The $z$-average diameter and PDI of S/O nanodispersions were measured shortly after preparation (Figure 2 ). The size variations demonstrated that the nanodispersed particle sizes closely correlated to the SE:BSA:HA molar ratio. The molar ratio between SE:BSA corresponding to $25 \mathrm{mg} / \mathrm{mL}$ of SE and $0.5 \mathrm{mg} / \mathrm{mL}$ of BSA led to the smallest nanodispersions, particularly for 15 , 50 , and $20 \leq M_{\mathrm{w}} \leq 50 \mathrm{kDa} \mathrm{HA}(160,115$, and $129 \mathrm{~nm}$, respectively). The best BSA:HA ratio obtained was 60:1 corresponding to $0.5 \mathrm{mg} / \mathrm{mL}$ of BSA with $0.006 \mathrm{mg} / \mathrm{mL}$ of HA which imparted a pronounced particle size reduction. The amount of BSA doubled compared to that without the presence of HA.

3.1.1. S/O Nanodispersion Stability Studies. The monitoring of $\mathrm{S} / \mathrm{O}$ nanodispersion production involves the study of their physical and biological stability. In order to evaluate physical stability of S/O nanodispersions their size distribution and polydispersity (PDI) was monitored immediately after production and for three months of storage at room temperature. The results shown in Figure 3 illustrate the combined simultaneous measurements of average diameter and PDI for the best nanodispersions for each HA tested. It seems clear that, after 2 months of storage, sample D displayed the lowest variation in terms of particle size, as well as of polydispersity. Other nanodispersions, especially $\mathrm{B}$ and $\mathrm{C}$, are not so stable during storage, as illustrated by the considerable vatiation in particle size (Figure 3). Sample D was the chosen nanoformulation for further experiments.

The biological stability of formulation $\mathrm{D}$ was measured in terms of lipid peroxidation during three weeks. As expected, no oxidation was verified at time zero and a low oxidation level was detected after one week $(3.3 \mathrm{mmol} / \mathrm{mL})$ and after three weeks of storage $(5.4 \mathrm{mmol} / \mathrm{mL}$ of $\mathrm{MDA})$, corresponding to a peroxidation level of $5.5 \%$. These results suggest that no additional antioxidant is required to prevent the production of free radicals. The nanodispersion itself does not contribute to the formation of oxidation products.

3.1.2. Production Yield and Assembly Efficiency of Nanodispersions. The production yield (PY) and the assembly efficiency $(\mathrm{AE})$ of $\mathrm{S} / \mathrm{O}$ nanodispersions were evaluated. Table 1 presents the particle size, polydispersity, production yield, and assembly efficiency for the formulations containing the different molecular weight HA. The assembly efficiency obtained was higher than $89.6 \%$, suggesting that almost all of the HA used in the preparation of S/O nanodispersion was actually entrapped/ adsorbed by the protein. The production yield was greater than $70.5 \%$.

The small size, low PDI, good stability, attractive price, and desirable performance adds value to nanodispersion $\mathrm{D}$ as a potential transdermal pharmaceutical-cosmetic product. Figure 4 illustrates spherical and homogeneous oil-based nanodispersion with $z$-average diameter of around $130 \mathrm{~nm}$ confirmed by TEM results.

3.2. Cytotoxicity Evaluation. The cytotoxicity of the most suitable nanodispersion produced was assessed in in vitro cultured fibroblasts and keratinocytes. In order to establish the effect of BSA mixed with HA nanodispersions on exposed BJ5ta and NCTC2544 cells (Figure 5), all tested conditions were evaluated after 24 and $48 \mathrm{~h}$. No toxicity was observed for the range from 0.5 to $2 \% \mathrm{v} / \mathrm{v}$ formulation, corresponding to the concentrations of.2.5 $-10 \mu \mathrm{g} / \mathrm{mL}$ for BSA and $0.03-0.12 \mu \mathrm{g} / \mathrm{mL}$ for $\mathrm{HA}$, thought keratinocytes were slightly more sensitive to the formulation.

3.3. In Vitro Permeation Studies of S/O Nanodispersion To Achieve the Ideal Formulation Concentration. Considering the previous best formulation (D) in terms of particle size and stability and its low toxicity for concentrations in the range of $2.5-10 \mu \mathrm{g} / \mathrm{mL}$ for BSA and $0.03-0.12 \mu \mathrm{g} / \mathrm{mL}$ of $\mathrm{HA}$, skin permeation studies were conducted. Quantitative estimation for the permeation approaches of BSA/HA nanodispersions were performed and are presented in Figure 6. It is

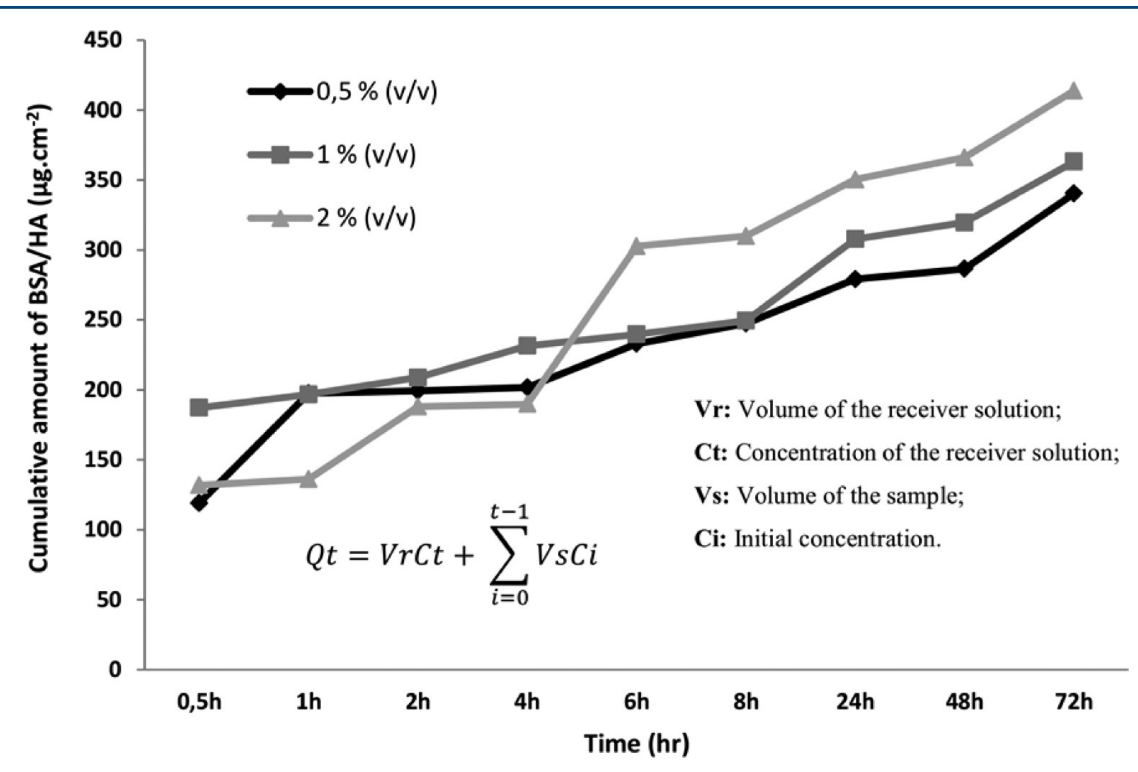

Figure 6. Permeation profile of S/O BSA/HA nanodispersions across abdominal pig skin. Formulations (a) $0.5 \%$ (v/v) corresponds to $2.5 \mu \mathrm{g} / \mathrm{mL}$ of BSA and $0.03 \mu \mathrm{g} / \mathrm{mL}$ of HA; (b) $1 \%(\mathrm{v} / \mathrm{v}$ ) corresponds to $5 \mu \mathrm{g} / \mathrm{mL}$ of BSA and $0.06 \mu \mathrm{g} / \mathrm{mL}$ of HA and c) $2 \%$ (v/v) corresponds to $10 \mu \mathrm{g} / \mathrm{mL}$ of BSA and $0.12 \mu \mathrm{g} / \mathrm{mL}$ of HA. Each point represent the mean $\pm \mathrm{SD}, n=3$. The data are expressed as the cumulative nanodispersion permeation (Qt) per unit of skin surface area, $\mathrm{Qt} / A\left(A=0.785 \mathrm{~cm}^{2}\right)$. 
clear from the graph that the cumulative amount of BSA/HA nanodispersion in the three concentrations exhibits an excellent performance over time, mainly from 6 to $72 \mathrm{~h}$. High cumulative amounts were achieved like in other successful studies that promote transdermal delivery of compounds through the skin. ${ }^{38-40}$ Sample with $2 \%(\mathrm{v} / \mathrm{v})$ presents the highest cumulative amount of permeated BSA/HA conjugate corresponding to greater skin diffusion. These results clearly demonstrate that the penetration of HA into the skin is mainly due to its lipophilic character, and an increase of the donor sample concentration, strengthening percutaneous permeation efficiency.

TEM images in donor (initial nanodispersion) and in receptor (final nanodispersion solution) compartments showed irregular nanodispersions of spherical integral small particles and disintegrated larger particles. Several factors including test duration, sampling frequency, sink condition, stirring rate, (a)

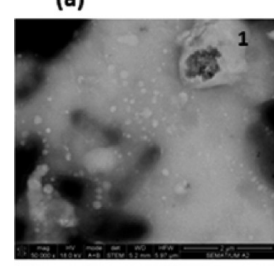

2

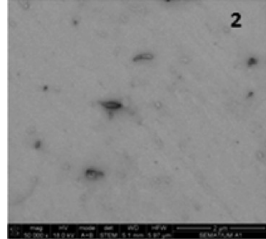

(b)
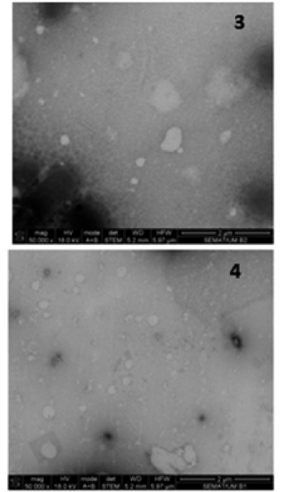

(c)

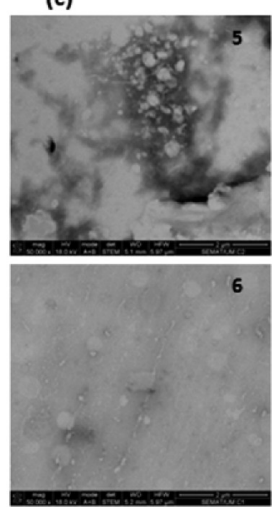

Figure 7. TEM microphotograph of BSA/HA nanodispersion of (a) $0.5 \% \mathrm{v} / \mathrm{v}$ formulation, (b) $1 \% \mathrm{v} / \mathrm{v}$ formulation, and (c) $2 \% \mathrm{v} / \mathrm{v}$ formulation, in liquid donor compartment $(1,3,5)$ and in liquid receptor compartment $(2,4,6)$, after $72 \mathrm{~h}$ of incubation at $37^{\circ} \mathrm{C}$. diffusion membrane material, and others may contribute to these irregularities and to the differences in the cumulative amount values. Furthermore, the initial solution was composed of large sized particles (Figure 7: a1, b3, and c5) that had a tendency to aggregate and disintegrate when forced to diffuse by gravity.

The percutaneous penetration mechanism was also qualitatively analyzed by histological examination of the skin. Fluorescent and confocal images of S/O BSA/HA nanodispersions are shown in Figures 8 and 9. Comparing both control sample and the corresponding fluorescence images of the formulations, fluorescence emitted by FITC and RITC provides clear evidence of percutaneous permeation and the successful permeation across the SC layer and deeper diffusion into the dermis.

The permeation profile of the BSA/HA nanodispersions through the layers of pig skin were investigated by confocal laser scanning microscopy. Confocal images in Figure 9 are divided into 2D images (FITC, RITC, and Merge) and 3D, the upper images (3D and a 3D close-up) showing penetration of BSA/HA through the pig skin. Penetration through stratum corneum is the rate limiting step in the overall transdermal delivery. Once the formulation crosses stratum corneum, its further penetration or absorption is relatively easy. ${ }^{41}$ Figures 9 clearly shows the penetration of the formulation through the different skin layers. The hydrophobic moiety of formulation successfully allowed the permeation of a hydrophilic compound (HA) through the mortar and brick structure of stratum corneum, naturally composed of corneocytes surrounded by lipid bilayers, resistant to hydrophilic molecules. Confocal images corroborate the previous assumption of formulation disintegration as it crosses the skin. It is possible to observe, especially in the dermis layer, high fluorescence of the dissociated compounds.

\section{DISCUSSION}

HA has been extensively explored as a drug carrier for target specific and long-acting delivery of protein, peptide, and

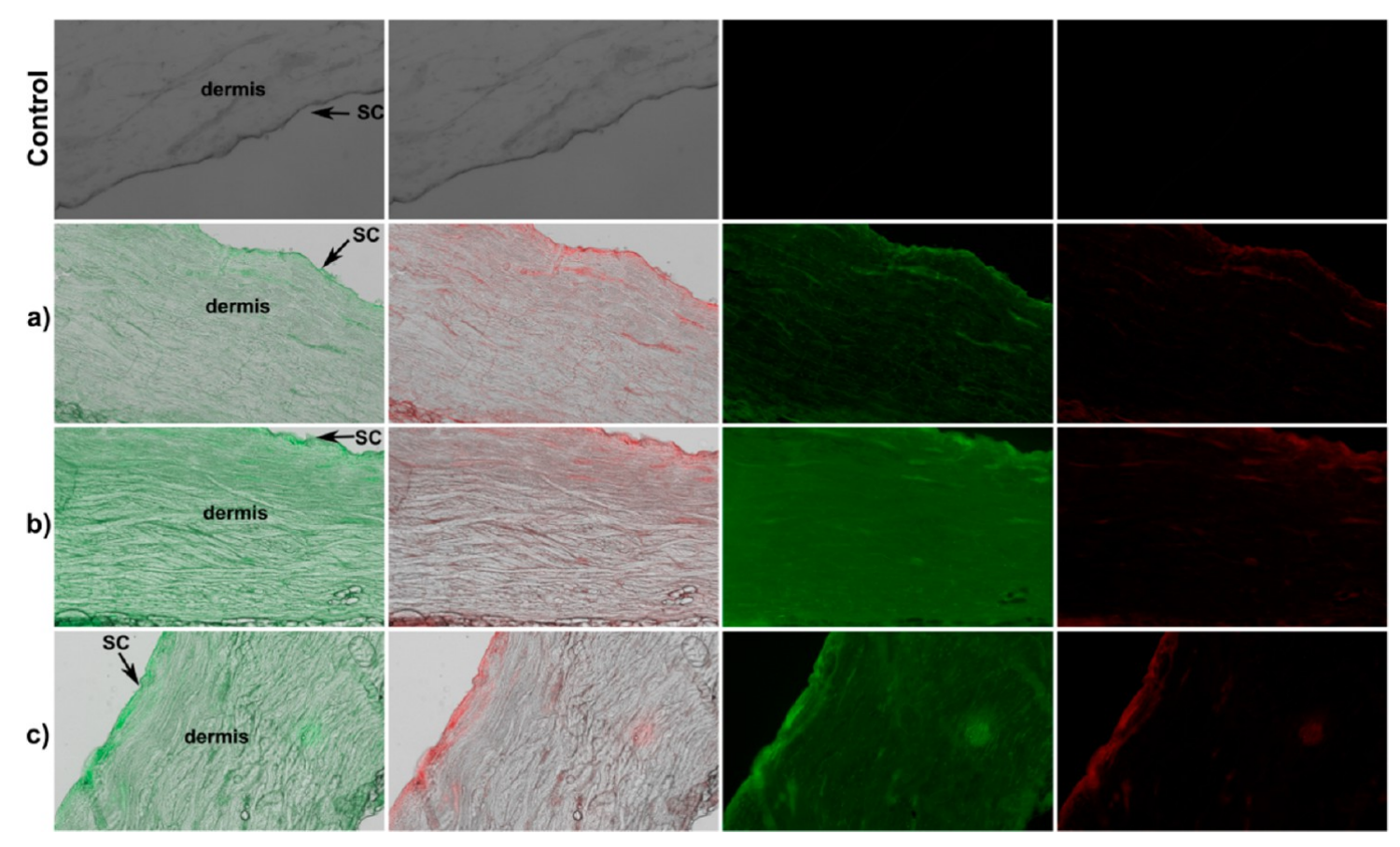

Figure 8. Fluorescent images showing the penetration of BSA nanodispersion with HA into pig skin. Penetration of S/O BSA/HA nanodispersion at (a) $0.5 \%$, (b) $1 \%$, and (c) $2 \% \mathrm{v} / \mathrm{v}$ formulation. Thumbnails $1,2,3$, and 4 (numbered left to right in each row) show the penetration of BSA-FITC at phase contrast with filter FITC, HA-RITC at phase contrast with filter RITC, BSA-FITC with filter FITC, and HA-RITC with filter RITC, respectively. 

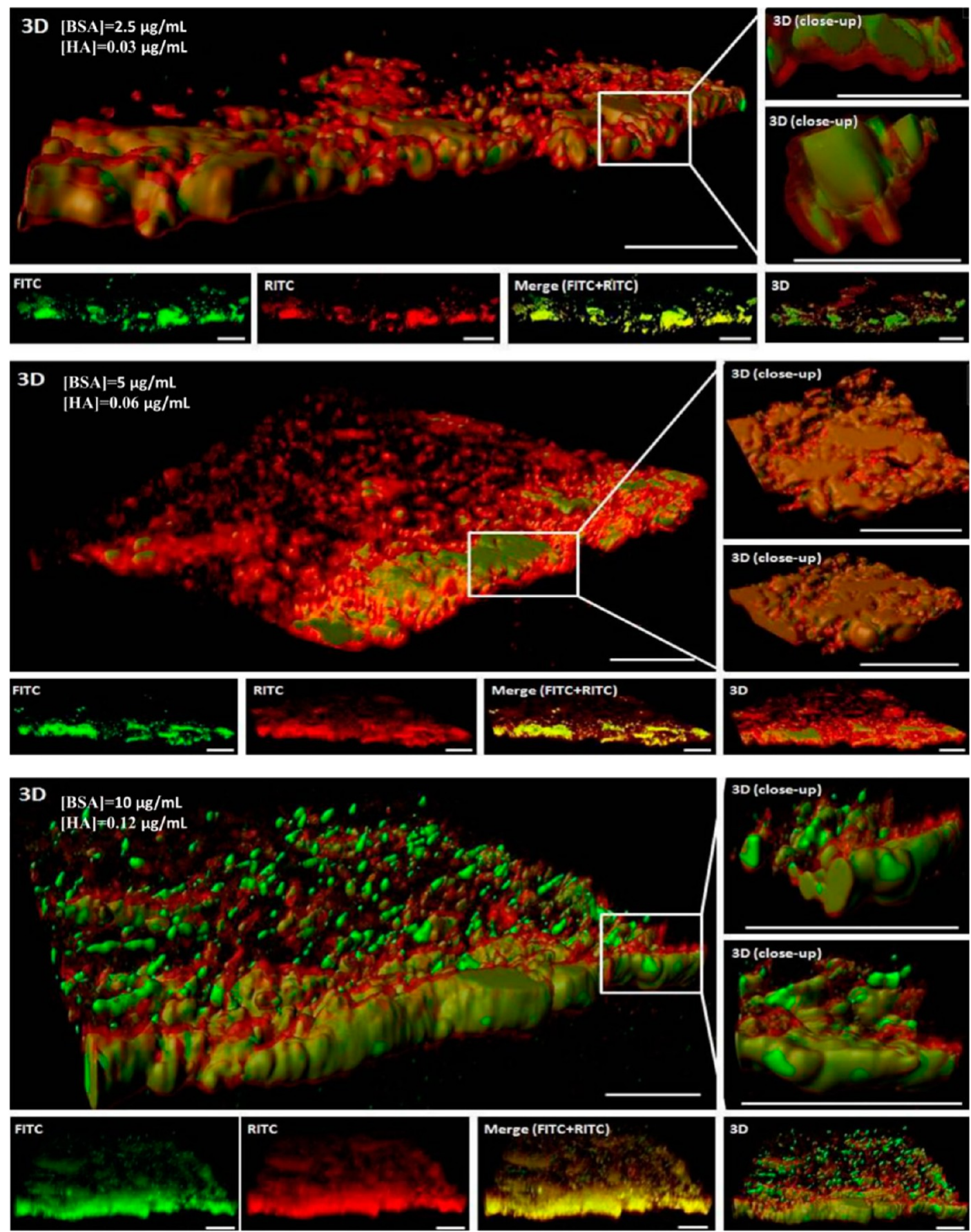

Figure 9. Confocal images of BSA/HA nanodispersions in epidermis and dermis pig skin. Bottom illustrations: FITC images correspond to the green color with emission maxima at $520 \mathrm{~nm}$ emitting from FITC labeled BSA protein, and RITC correspond to the red color with emission maxima at $595 \mathrm{~nm}$ emitting from RITC labeled HA. The Merge images (FITC + RITC) combine green and red fluorescent signals emitted from the same sample. Upper: 3D illustration combines green and red channels (FITC-BSA green and RITC-HA red channel), and the yellowish color symbolizes the overlapping of both. The right 3D (close-up) image demonstrates the view of the skin piece in detail where the penetration of FITC-BSA and HA-RICT is easily detected.

nucleotide therapeutics. ${ }^{13}$ Although there is no study available using HA as nanodispersion for transdermal transport system, this constitutes a surplus value for the pharmaceutical/cosmetic area. HA is a powerful antioxidant, helping natural defenses and keeping the skin youthful, hydrated, and toned. In previous work we successfully demonstrated the possibility of delivering large proteins (BSA) transdermally for a range of medical applications. ${ }^{26}$ Herein, we incorporated HA into the BSA nanodispersion and the results demonstrated that the presence of HA does not unsettle the final nanodispersion. In addition, with the presence of HA a lesser amount of protein is required which contributes for a lower $z$-average diameter of the final particle. The association of BSA with HA was crucial because its presence prevented the emulsion instability (data not shown). A similar addition was used by Piao et al. to prevent the release of sodium diclofenac into the $\mathrm{S} / \mathrm{O}$ suspension. ${ }^{42}$ The formulation optimization was conducted by study of a wide range of HA molecular weights at different conditions. An optimum molar ratio among SE:BSA:HA in the 
complex was found. The results demonstrated that nanodispersions from lower molecular weight HA displayed the smaller particle sizes. This is mainly due to the low molecular weight of HA allowing small extent of polymerization and gives rise to short carbohydrate chains of small molecular size, which render high chain flexibility to assemble, small three-dimensional structures, and weak steric interference, favoring the formation of a compact assembly of BSA/HA. ${ }^{14}$ The collective results revealed that nanodispersions were stable over three months in terms of physical and chemical stability. These results were expectable because BSA/ HA molecules were coated with hydrophobic surfactant, which leads to stronger hydrophobicity enhancing their dispersion into the oil phase and boosting compactness of the final S/O BSA/HA nanodispersion. Moreover, it was reported that a typical $\mathrm{S} / \mathrm{O}$ nanodispersion can be prepared with low viscosity of the system resulting from the low surfactant concentration. ${ }^{25}$ The stability of $\mathrm{S} / \mathrm{O}$ nanodispersion is sufficiently high to prevent precipitation or particle size changes for up to one month. ${ }^{43}$

Reinforcing the good results obtained in terms of particle size, stability, and attractive price, the developed S/O BSA/HA nanodispersion does not present toxicity in the range of $2.5-10 \mu \mathrm{g} / \mathrm{mL}$ for BSA and $0.03-0.12 \mu \mathrm{g} / \mathrm{mL}$ of $\mathrm{HA}$. The concentration range selected constitutes the composition for designing a BSA/HA formulation as potential transdermal delivery for biopharmaceutical demands. In these conditions, S/O BSA/HA nanodispersions are able to reach the lipid skin layers and the presence of HA can be detected in the deeper layers of the skin.

The successful permeation through the skin was evaluated by quantitative and qualitative estimation. The results suggest that BSA/HA nanodispersion disintegrates as it crosses the stratum corneum into the dermis. HA has a low molecular weight and was 60× (molar ratio BSA/HA 60:1) less concentrated comparatively with BSA, making it more effective to diffuse deeper into the skin layer. Thus, as the S/O BSA/HA nanodispersion permeates the skin and the BSA could be hydrolyzed by proteases residing in the medium, the HA became more available and deeply diffuse into the skin. These factors would increase its availability, accessibility, and activity to act on the desired skin targets.

\section{CONCLUSIONS}

BSA/HA S/O nanodispersion production was successfully achieved by a two phase high energy methodology. The quantitative and qualitative in vitro permeation results successfully demonstrated the effective penetration of BSA/HA nanodispersion to the epidermis and even to the dermis of pig skin. These results reveal the feasibility of using S/O nanodispersions of BSA/HA as a model system for the improvement of skin biomechanical properties and maintenance of an optimal hydration level and firmness. Moreover, transdermal hyaluronic acid could be a stimulating way to replenish HA in the dermis inexpensively and without the discomfort associated with injectables.

\section{AUTHOR INFORMATION}

\section{Corresponding Author}

*Tel: +351 253604 400. Fax: +351 253604 429. E-mail: artur@ deb.uminho.pt.

\section{Present Address}

${ }^{\perp}$ (C. S.) Biomaterials, Biodegradables and Biomimetics, Dept. of Polymer Engineering, University of Minho AvePark, Zona
Industrial da Gandra, S. Claudio do Barco 4806-909 Caldas das Taipas, Guimaraes, Portugal.

\section{Notes}

The authors declare no competing financial interest.

\section{ACKNOWLEDGMENTS}

We thank Matadouro Central Carnes de Entre Douro e Minho, Lda, for their support on pig samples. The histological studies were supported by the Department of Histology from Life and Health Sciences Research Institute (ICVS), University of Minho. The confocal 3D images were performed by Department of Chemistry and Kanbar Laboratory for Nanomaterials Bar-Ilan University Center for Advanced Materials and Nanotechnology. C.S. would like to thank Fundação para a Ciência e a Tecnologia for their funding (SFRH/BPD/46515/2008). T.M. would like to thank Fundação para a Ciência e a Tecnologia for their funding (SFRH/BPD/47555/2008).

\section{REFERENCES}

(1) Schaefer, H.; Redelmeier, T. E. Skin barrier: principles of percutaneous absorption; Karger: Basel, 1996; pp 1-310.

(2) Bensouilah, J.; Buck, P. Aromadermatology: Aromatherapy in the Treatment and Care of Common Skin Conditions; Radcliffe: Seattle, 2006; pp $1-11$.

(3) Trommer, H.; Wartewig, S.; Böttcher, R.; Pöppl, A.; Hoentsch, J.; Ozegowski, J. H.; Neubert, R. H. H. The effects of hyaluronan and its fragments on lipid models exposed to UV irradiation. Int. J. Pharm. 2003, 254 (2), 223-234.

(4) Bergeret-Galley, C.; Latouche, X.; Illouz, Y.-G. The Value of a New Filler Material in Corrective and Cosmetic Surgery: DermaLive and DermaDeep. Aesthetic Plast. Surg. 2001, 25 (4), 249-255.

(5) Duranti, F.; Salti, G.; Bovani, B.; Calandra, M.; Rosati, M. L. Injectable hyaluronic acid gel for soft tissue augmentation. A clinical and histological study. Dermatol. Surg. 1998, 12, 1317-25.

(6) Narins, R. S.; Brandt, F.; Leyden, J.; Lorenc, Z. P.; Rubin, M.; Smith, S. A Randomized, Double-Blind, Multicenter Comparison of the Efficacy and Tolerability of Restylane Versus Zyplast for the Correction of Nasolabial Folds. Dermatol. Surg. 2003, 29 (6), 588-595.

(7) Laurent, T. C. Structure of hyaluronic acid. Chem. Mol. Biol. Intracell. Matrix 1970, 703-732.

(8) Kablik, J.; Monheit, G. D.; Yu, L.; Chang, G.; Gershkovich, J. Comparative Physical Properties of Hyaluronic Acid Dermal Fillers. Dermatol. Surg. 2009, 35, 302-312.

(9) Milas, M.; Rinaudo, M.; Roure, I.; Al-Assaf, S.; Phillips, G. O.; Williams, P. A. Comparative rheological behavior of hyaluronan from bacterial and animal sources with cross-linked hyaluronan (Hylan) in aqueous solution. Biopolymers 2001, 59 (4), 191-204.

(10) Tengblad, A.; Laurent, U. B.; Lilja, K.; Cahill, R. N.; EngströmLaurent, A.; Fraser, J. R.; Hansson, H. E.; Laurent, T. C. Concentration and relative molecular mass of hyaluronate in lymph and blood. Biochem. J. 1986, 236, 521-525.

(11) Garg, H. G.; Hales, C. A. Chemistry and biology of hyaluronan; Elsevier Science \& Technology Books: London, 2004.

(12) Brown, M. B.; Jones, S. A. Hyaluronic acid: a unique topical vehicle for the localized delivery of drugs to the skin. J. Eur. Acad. Dermatol. Venereol. 2005, 19, 308-318.

(13) Oh, E. J.; Park, K.; Kim, K. S.; Kim, J.; Yang, J.-A.; Kong, J.-H.; Lee, M. Y.; Hoffman, A. S.; Hahn, S. K. Target specific and long-acting delivery of protein, peptide, and nucleotide therapeutics using hyaluronic acid derivatives. J. Controlled Release 2010, 141 (1), 2-12.

(14) Kong, M.; Chen, X.; Park, H. Design and investigation of nanoemulsified carrier based on amphiphile-modified hyaluronic acid. Carbohydr. Polym. 2011, 83 (2), 462-469.

(15) King, M. J.; Badea, I.; Solomon, J.; Kumar, P.; Gaspar, J. K.; Foldvari, M. Transdermal Delivery of Insulin from a Novel Biphasic Lipid System in Diabetic Rats. Diabetes Technol. Ther. 2002, 4 (4), 479488. 
(16) Glenn, G. M.; Kenney, R. T. Mass Vaccination: Solutions in the Skin. In Mass Vaccination: Global Aspects-Progress and Obstacles; Plotkin, S., Ed.; Springer: Berlin Heidelberg, 2006; Vol. 304, pp 247268.

(17) Prausnitz, M. R.; Langer, R. Transdermal drug delivery. Nat. Biotechnol. 2008, 1261-1268.

(18) Cevc, G.; Vierl, U. Nanotechnology and the transdermal route: A state of the art review and critical appraisal. J. Controlled Release 2010, 141 (3), 277-299.

(19) Karande, P.; Jain, A.; Ergun, K.; Kispersky, V.; Mitragotri, S. Design principles of chemical penetration enhancers for transdermal drug delivery. Proc. Natl. Acad. Sci. U.S.A. 2005, 102 (13), 4688-4693.

(20) Scheuplein, R. J.; Blank, I. H. Permeability of the skin. Physiol. Rev. 1971, 51 (4), 702-747.

(21) Bos, J. D.; Meinardi, M. M. H. M. The 500 Da rule for the skin penetration of chemical compounds and drugs. Exp. Dermatol. 2000, 9 (3), 165-169.

(22) Elias, P. M. Epidermal lipids, barrier function, and desquamation. J. Invest. Dermatol. 1983, 80, 44-49.

(23) Bouwstra, J. A.; Gooris, G. S.; Bras, W.; Downing, D. T. Lipid organization in pig stratum corneum. J. Lipid Res. 1995, 36 (4), 685-95.

(24) Wille, J. J. Skin Delivery Systems: Transdermals, Dermatologicals, and Cosmetic Actives; Blackwell: 2006; p 392.

(25) Tahara, Y.; Kamiya, N.; Goto, M. Solid-in-oil dispersion: A novel core technology for drug delivery systems. Int. J. Pharm. 2012, 438 (12), 249-257.

(26) Martins, M.; Azoia, N. G.; Ribeiro, A.; Shimanovich, U.; Silva, C.; Cavaco-Paulo, A. In vitro and computational studies of transdermal perfusion of nanoformulations containing a large molecular weight protein. Colloids Surf., B 2013, 108, 271-278.

(27) Walsh, G. Biopharmaceutical benchmarks 2010. Nat. Biotechnol. 2010, 28, 917-924.

(28) Price, R. D.; Berry, M. G.; Navsaria, H. A. Hyaluronic acid: the scientific and clinical evidence. J. Plast., Reconstr., Aesthetic Surg. 2007, 60 (10), 1110-1119.

(29) Kong, M.; Park, H. J. Stability investigation of hyaluronic acid based nanoemulsion and its potential as transdermal carrier. Carbohydr. Polym. 2011, 83 (3), 1303-1310.

(30) Lowry, O. H.; Rosebrough, N. J.; Farr, A. L.; Randall, R. J. Protein measurement with the Folin phenol reagent. J. Biol. Chem. 1951, 193 (1), 265-275.

(31) Ohkawa, H.; Ohishi, N.; Yagi, K. Assay for lipid peroxides in animal tissues by thiobarbituric acid reaction. Anal. Biochem. 1979, 95, 351-358.

(32) Tim, M. Rapid colorimetric assay for cellular growth and survival: Application to proliferation and cytotoxicity assays. J. Immunol. Methods 1983, 65 (1-2), 55-63.

(33) Edmondson, J. M.; Armstrong, L. S.; Martinez, A. O. A rapid and simple MTT-based spectrophotometric assay for determining drug sensitivity in monolayer cultures. Methods Cell Sci. 1988, 11 (1), 15-17.

(34) Mosmann, T. Rapid colorimetric assay for cellular growth and survival: application to proliferation and cytotoxicity assays. J. Immunol. Methods 1983, 65, 55-63.

(35) Meerloo, J. V.; Kaspers, G. J.; Cloos, J. Cell sensitivity assays: the MTT assay. Methods Mol. Biol. 2011, 731, 237-245.

(36) Berridge, M. V.; Herst, P. M.; Tan, A. S. Tetrazolium dyes as tools in cell biology: new insights into their cellular reduction. Biotechnol. Annu. Rev. 2005, 11, 127-152.

(37) Stockert, J. C.; Blázquez-Castro, A.; Cañete, M.; Horobin, R. W.; Villanueva, Á. MTT assay for cell viability: Intracellular localization of the formazan product is in lipid droplets. Acta Histochem. 2012, 114 (8), 785-796.

(38) ElMeshad, A.; Tadros, M. Transdermal Delivery of an AntiCancer Drug via W/O Emulsions Based on Alkyl Polyglycosides and Lecithin: Design, Characterization, and In Vivo Evaluation of the Possible Irritation Potential in Rats. AAPS PharmSciTech 2011, 12 (1), $1-9$.

(39) Shah, S.; Tahir, M.; Safdar, A.; Riaz, R.; Shahzad, Y.; Rabbani, M.; Karim, S.; Murtaza, G. Effect of Permeation Enhancers on the Release
Behavior and Permeation Kinetics of Novel Tramadol Lotions. Trop. J. Pharm. Res. 2013, 12 (1), 27-32.

(40) Ghanbarzadeh, S.; Arami, S. Enhanced Transdermal Delivery of Diclofenac Sodium via Conventional Liposomes, Ethosomes, and Transfersomes. BioMed Res. Int. 2013, 2013, 7.

(41) Chourasia, M. K.; Kang, L.; Chan, S. Y. Nanosized ethosomes bearing ketoprofen for improved transdermal delivery. Results Pharma Sci. 2011, 1 (1), 60-67.

(42) Piao, H.; Kamiya, N.; Watanabe, J.; Yokoyama, H.; Hirata, A.; Fujii, T.; Shimizu, I.; Ito, S.; Goto, M. Oral delivery of diclofenac sodium using a novel solid-in-oil suspension. Int. J. Pharm. 2006, 313 (1-2), $159-162$.

(43) Tahara, Y.; Honda, S.; Kamiya, N.; Piao, H.; Hirata, A.; Hayakawa, E.; Fujii, T.; Goto, M. A solid-in-oil nanodispersion for transcutaneous protein delivery. J. Controlled Release 2008, 131 (1), 14-18. 